\title{
Disrupting the isochrony underlying rhythm: An asymmetry in discrimination
}

\author{
JAMSHED JAY BHARUCHA and JOHN H. PRYOR \\ Dartmouth College, Hanover, New Hampshire
}

\begin{abstract}
Recognition memory for equitone sequences was tested as a function of whether the pattern of stimulus onsets mapped easily onto an isochronous grid (rhythmic) or did not (disrupted). In a same-different task, discrimination between a rhythmic sequence and its disrupted variant was better when the rhythmic sequence was presented first and the disrupted variant second than vice versa. Analogous effects have been obtained for melodies and chord sequences as a function of tonal structure, and for text as a function of semantic and pragmatic coherence. In each domain, discrimination between coherent sequences and anomalous variants is better when the coherent version is presented first.
\end{abstract}

A sequence of tones is perceptually organized with reference to an isochronous pulse or grid (Povel, 1984), typically heard as a beat. The listener extracts from a sequence the most economical isochronous grid, one that maps onto as many stimulus onsets as possible (Povel, 1984). Sequences are more accurately imitated if the onsets map onto an isochronous grid than if they are selected at random, and imitations of the latter are biased in the direction of the former (Fraisse, 1982). Although a grid of sufficiently fine resolution can always be found that maps onto all the stimulus onsets, stimulus onset asynchronies (SOAs) of substantially less than 150 to $200 \mathrm{msec}$ cease to provide a beat (Fraisse, 1982; Handel \& Lawson, 1983; Povel, 1984). The grid need not be as fine as the smallest SOA in a sequence; onsets may fall between the cracks if they are simple subdivisions of the grid interval (see Povel, 1984). In the present paper, a sequence of tones will be called "rhythmic" if its onsets map onto an isochronous grid, and "disrupted" if not. ${ }^{1}$

Rhythm is one of several domains in which we have intuitions of coherence and anomaly for sequences of events. Intuitions of coherence are driven by cognitive structures and processes which organize information into recognizable and manageable units. Rhythmic coherence derives to a large extent from isochrony. The special properties of isochrony have been noted in the context of both music (Deutsch, 1983; Fraisse, 1982; Handel \& Lawson, 1983; Jones, Kidd, \& Wetzel, 1981; Lerdahl \& Jackendoff, 1983; Povel, 1984) and speech (Fowler, 1983). Melodic and harmonic coherence stem from, among other things, constraints on the selection of tones (Castellano, Bharucha, \& Krumhansl, 1984; Cuddy, Cohen, \& Miller, 1979; Dowling, 1978; Krumhansl, 1979)

This research was supported in part by a Biomedical Research Support Grant from the Department of Health and Human Services (2-S07RR07056-09). The authors thank Jack Baird, James Bartlett, Jay Dowling, and Caroline Palmer for helpful discussions. Requests for reprints should be sent to the first author, Department of Psychology, Dartmouth College, Hanover, NH 03755. and chords (Bharucha \& Krumhansl, 1983) and constraints on temporal order (Bharucha, 1984a). Textual coherence hinges on the use of familiar settings, referents, causal relations, and motivations (Black, 1984). Text that cannot be organized in terms of entrenched higher order cognitive units is considered to be anomalous, and may, at retrieval, be forced into a familiar organization (F. C. Bartlett, 1932). In each case, intuitions of coherence result from the subsumption of a sequence by more abstract organizing units in hierarchical fashion.

Our intuitions of coherence and anomaly thus cut across domains. The existence of processes common to these domains is borne out by similar advantages that accrue to coherent sequences across domains. Evidence in other domains includes an increased perceptibility of coherent over anomalous sentences (Miller \& Isard, 1963), superior memory for scripted text over scrambled text (Bower, Black, \& Turner, 1979), a bias toward coherence in the recall of anomalous text (F. C. Bartlett, 1932), superior memory for objects embedded in typical versus scrambled street scenes (Biederman, Glass, \& Stacy, 1973), and superior memory for tonal over atonal melodies (Francès, 1972).

If subjects are presented with a sequence in which all the elements conform to a coherent structure, a subsequent recognition test reveals that new items which conform to the coherent structure are more easily confused with the original than are new items which violate the structure. This is true for tones embedded in diatonic melodies (Bharucha, 1984a; Cuddy et al., 1979; Dowling, 1978), chords embedded in diatonic chord sequences (Bharucha \& Krumhansl, 1983; Krumhansl, Bharucha, \& Castellano, 1982), and words embedded in coherent text (Bower et al., 1979; Graesser, Gordon, \& Sawyer, 1979).

A corollary principle that seems to cut across domains is an asymmetry in the detection of alterations to a sequence, as a function of whether coherence is violated or established. Dowling and Bartlett (1981; see J. C. Bartlett, 1984) presented subjects with two melodies in suc- 
cession, one completely diatonic and the other either identical or with one tone replaced by a nondiatonic tone. They varied the order of presentation, and found that melodies were judged more dissimilar in a rating task, and were more accurately discriminated in a same-different task, when the nondiatonic tone occurred in the second sequence rather than in the first.

Bharucha (1984a) used melodies that clearly implied a single chord. Altered melodies were constructed by replacing a tone with one that disrupted the chordal implication. The two melodies were more accurately discriminated from each other when the chordal melody occurred first and the altered melody second than vice versa. In general, then, two predominantly tonal melodies, one containing a disruptive tone, are more accurately discriminated from each other when the one with the disruptive tone is heard last.

This asymmetry has also been found for sequences of chords. If a sequence of diatonic chords (all drawn from the same key) is altered by replacing one chord with a nondiatonic chord, the two sequences are more accurately discriminated if the disrupted version follows the completely diatonic version than vice versa (Bharucha \& Krumhansl, 1983).

Bharucha, Olney, and Schnurr (1985) found an analogous effect using linguistic materials. The analog of diatonicity was semantic or pragmatic coherence. Consider the following pair of sentences:

The ship pitched wildly in the storm.

John hoped the weather would clear up.

If "weather" is replaced with "acne," the coherent vignette is rendered anomalous:

The ship pitched wildly in the storm.

John hoped the acne would clear up.

Subjects were presented with 40 sentence pairs during a study phase and were later tested with each pair either in identical form or with one word changed, thereby changing the meaning of one of the sentences. Discrimination accuracy was better when a coherent pair was studied and its anomalous variant used as the test item than vice versa.

Two general conclusions about underlying structures and processes are suggested by these asymmetries. First, the matching of the test sequence with the memory representation of the original sequence is not symmetric. Each element of the test sequence is checked for inclusion in the memory representation of the original sequence, but not vice versa. This results in better detection of additions of an anomaly than of omissions of an anomaly.

Second, the representation of a coherent sequence includes information about more than just the individual elements. The representation of tonal melodies and chord sequences includes abstract tonal structures such as chord functions and keys, since listeners abstract chord functions from unaccompanied tonal melodies (Bharucha, 1984b), and abstract keys from sequences of chord functions (Krumhansl \& Kessler, 1982). New elements from the same chord function or key are easily confused with the original because they match the memory representation at the level of chord function or key (Bharucha, 1984a; Bharucha \& Krumhansl, 1983). New elements from different chord functions or keys do not match any level of the memory representation of the original. This view is consistent with formal theories that represent musical sequences as temporal hierarchies (Deutsch \& Feroe, 1981; Lerdahl \& Jackendoff, 1983; Schenker, 1979). The representation of coherent text includes abstract structures such as plots (Lehnert, 1981), episodes (Rumelhart, 1977), or scripts (Bower et al., 1979; Graesser et al., 1979). New elements that match abstract levels of the memory representation are more easily confused with the original than are new elements that do not match at any level (Bower et al., 1979; Graesser et al., 1979). This view is consistent with the hierarchical structures proposed by most accounts of discourse (Black, 1984).

In this study, we predicted an analogous asymmetry for rhythmic sequences. By most accounts, rhythmic sequences are represented hierarchically (Deutsch, 1983). Povel's (1984) grid, with its subdivisions, is essentially hierarchical. Higher levels of hierarchical organization occur when the isochronous grid is metrically differentiated by means of acoustic accentuation or other cues (see Handel \& Lawson, 1983). On the basis of the asymmetries described above, we predicted that two sequences, one rhythmic and the other identical with the exception of one disrupted SOA, would be more easily discriminated if the disrupted sequence followed the rhythmic one than vice versa.

Figure 1 (top) shows a rhythmic sequence followed by a disrupted version resulting from lengthening one SOA

\section{STANDARD COMPARISON}
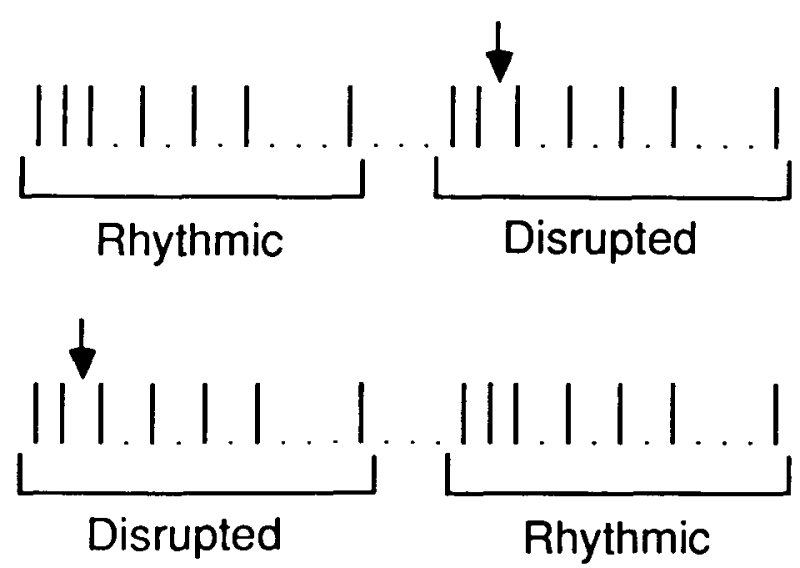

Figure 1. (Top) An example of a rhythmic sequence followed immediately by a disrupted variant. (Bottom) $A$ disrupted sequence followed immediately by a rhythmic variant. Vertical lines in the sequences represent tone onsets, and dots represent isochronous time slots of silence, using Povel's (1984) notation. The arrow indicates the position of the anomaly, a lengthening of one SOA. 
as indicated by the arrow. The same sequences presented in the reverse order are shown in the bottom panel. The vertical lines represent onsets of tones of equal duration, and the dots represent isochronous onset slots to which no tones have been assigned. This notation is adapted from Povel (1984).

The rhythmic sequences were constructed by distributing seven tones over 13 isochronous slots, such that a tone always occurred in the first and last slots. The interval between the last tone of the standard sequence and the first tone of the comparison sequence was the equivalent of three slots. This arrangement permitted the implication of a duple meter without interruption between the standard and the comparison; 16 isochronous slots would be heard as two groups of 8 , each of which would be heard as two groups of 4 , and so on. This particular parsing was in no way crucial to the experiment, but the use of a power of 2 as the number of slots merely maximized the likelihood that the sequence would be heard as rhythmically coherent, given the otherwise unmusical character of the stimuli. A tone always occurred in either the second or third slot, or in both, in order to establish isochrony early in the sequence.

\section{METHOD}

\section{Subjects}

Fifteen Dartmouth undergraduates in the introductory psychology course volunteered as subjects. Prior musical training, measured by the total number of instrument-years and voice-years of music lessons, ranged from 0 to 14 years, with an average of 4.9 years. All subjects reported normal hearing.

\section{Apparatus}

The subjects were tested individually on an IBM-XT microcomputer.

\section{Stimuli}

Eighteen sequences of seven tones each were constructed as follows. Tones were assigned to 7 of 13 isochronous slots with the following constraints: (1) tones were always assigned to the first and last slots, (2) no more than 3 slots in a row were assigned tones and no more than 4 slots in a row were left unassigned, in order to avoid long gaps, and (3) the second tone of the sequence was assigned to either the second or third slot. Within these constraints, the tones were assigned at random. Figure 1 shows a sequence generated in this manner.

All tones were of the same intensity, set at a comfortable level by the subject in advance of the experiment. All tones were of the same timbre (square waves). All tones within a trial were of the same duration, equal to $3 / 4$ of the slot interval. Pitch was constant over the standard sequence, then jumped to the octave above for the comparison sequence and remained constant until the end. For each trial, the pitch for the standard was chosen at random from the 12 equally tempered chromatic tones in the range of $220-415.3 \mathrm{~Hz}$. Tempo varied randomly from trial to trial within the range of 200-300 msec per slot-interval. The SOA thus ranged from $200 \mathrm{msec}$ (for tones in adjacent slots, in the fastest tempo) to $1,200 \mathrm{msec}$ (for tones with 4 intervening slots, in the slowest tempo). This range is within the range-from $125 \mathrm{msec}$ to 1,500 or $2,000 \mathrm{msec}$-normally used for the construction of rhythm (Povel, 1984), and is within the range-200 to $1,400 \mathrm{msec}-$ of spon- taneous tapping (Fraisse, 1982). The variation in tempo from trial to trial was designed to force a fresh parsing on each trial.

For each of the 18 sequences generated in this way (called rhythmic sequences), a disrupted sequence was generated by inserting a pause between two tones (the target SOA), the target position being selected at random. The duration of the pause was equivalent to half the duration of a slot interval.

On each trial, the subjects heard one of the 36 sequences (the standard sequence) followed by another sequence (the comparison sequence) that was either the same or different. This yielded 72 trials, 18 same trials with a rhythmic sequence, 18 same trials with a disrupted sequence, 18 different trials with a rhythmic sequence as the standard and its disrupted version as the comparison, and 18 different trials with a disrupted sequence as the standard and its rhythmic version as the comparison.

\section{Procedure}

A trial was initiated by the appearance of the trial number on the screen. Two seconds later, the standard and comparison sequences were heard in succession, separated by a pause equivalent to 3 time slots, as in Figure 1. The comparison sequence was played an octave higher than the standard, to clearly demarcate the two sequences. In addition, the letter $\mathrm{A}$ was displayed during the standard sequences, and the letter B during the comparison. The subjects had $4 \mathrm{sec}$ between trials in which to record their responses.

The instructions to the subjects were as follows: "On each trial you will hear a sequence of seven tones followed immediately by another sequence of seven tones in a higher octave. Your task is to decide whether they are different. When the two sequences are different, the timing of the tones will have been slightly altered." The subjects indicated their responses by selecting one of the following options: same, confident; same, not so confident; different, not so confident; different, confident. The responses were recorded by circling SS, S, D, or DD (representing the four options, respectively) on a response sheet.

The trials were presented in random order. Five practice trials preceded the experimental session. Each subject filled out a musicalbackground questionnaire after completing the experiment.

\section{RESULTS}

There were no discernible individual differences on the basis of musical background. The number of instrumentyears and voice-years of music lessons correlated at the $r=-.08$ level with proportion correct.

Responses were scored on a scale from 1 to $4(1=$ same, confident; $2=$ same, not so confident; $3=$ different, not so confident; 4 = different, confident). The mean response is shown in Table 1 for the two different and two same conditions. Treating both subjects and items as random factors, the mean response on different trials was significantly higher in the rhythmic-disrupted condition

Table 1

Discrimination Accuracy Between Rhythmic Sequences and

Disrupted Variants as a Function of Presentation Order

\begin{tabular}{lccccc}
\hline & \multicolumn{2}{c}{ Different } & & \multicolumn{2}{c}{ Same } \\
\cline { 2 - 3 } \cline { 5 - 6 } & $\begin{array}{l}\text { Rhythmic- } \\
\text { Disrupted }\end{array}$ & $\begin{array}{c}\text { Disrupted- } \\
\text { Rhythmic }\end{array}$ & Rhythmic & Disrupted \\
\hline Mean Response* & 2.54 & 2.27 & & 1.74 & 2.05 \\
Proportion Correct & .520 & .407 & & .807 & .689 \\
Area under ROC & .697 & .570 & & \\
\hline
\end{tabular}

${ }^{* 1}$ = same, confident; 4 = different, confident. 
(2.54) than in the disrupted-rhythmic condition (2.27) [quasi- $F^{\prime \prime}(1,27)=4.66, p<.05$; Winer, 1971]. The subjects were therefore more confident that the two sequences were different when the rhythmic version was presented first than when the disrupted version was presented first.

The mean response on same trials was significantly lower for rhythmic sequences (1.74) than for disrupted sequences $(2.05)$ [quasi- $F^{\prime \prime}(1,30)=4.86, p<.05$ ]. The subjects were therefore more confident of the identity of the rhythmic sequences than of the disrupted ones. For convenience, Table 1 also shows the mean proportion of correct responses, which was computed by collapsing over the two levels of confidence.

To test the possibility that the difference between rhythmic-disrupted and disrupted-rhythmic was due only to a bias to respond "same" when the last sequence was rhythmic, areas under receiver operating characteristic (ROC) curves were computed using hit and false-alarm rates for each of the three response criteria. The mean ROC area was significantly higher when the rhythmic version was presented first (.697) than when the disrupted version was presented first $(.570)[F(1,14)=16.23$, $p<.01$, thereby eliminating response bias as an explanation. The subjects were thus more sensitive to the difference between rhythmic sequences and disrupted variants when the former were heard first. Alternatively, rhythmic sequences were better recognized among disrupted distractors than disrupted sequences were among rhythmic distractors.

Length of the initial SOA did not affect performance. The difference in mean response between an initial SOA of one versus two slot intervals was insignificant for both different trials (quasi- $F^{\prime \prime}<1$ ) and same trials [quasi$\left.F^{\prime \prime}(1,25)=1.60, p=.2\right]$, and there were no interactions between length of initial SOA and either presentation order (on different trials) or rhythmicity (on same trials). There was no relationship between tempo and performance $(r=-.07)$, which is not surprising, given that tempo varied within a narrow window that reflected common usage and spontaneous rhythmic performance.

Items were classified according to the number of slots in the target SOA. There were 9,2, and 7 items with 1-, 2-, and 3-slot target SOAs, respectively (these numbers were not equal because the target interval was distributed randomly across items). The 2- and 3-slot target SOAs were combined to compute areas under ROC curves. Accuracy was significantly greater with 1 -slot $(.725)$ than with 2- or 3-slot $(.542)$ target SOAs $[F(1,14)=15.65$, $p<.01]$ and did not interact with presentation order. Thus, accuracy decreased as the size of the target SOA increased. Figure 2 shows performance as a function of target SOA, for the two presentation orders, using proportion of correct responses (an ROC value would not be meaningful for only two items with a 2-slot target SOA). This decreasing function is consistent with Weber's law; the dectectability of a constant change in SOA is a function of the absolute size of the SOA.

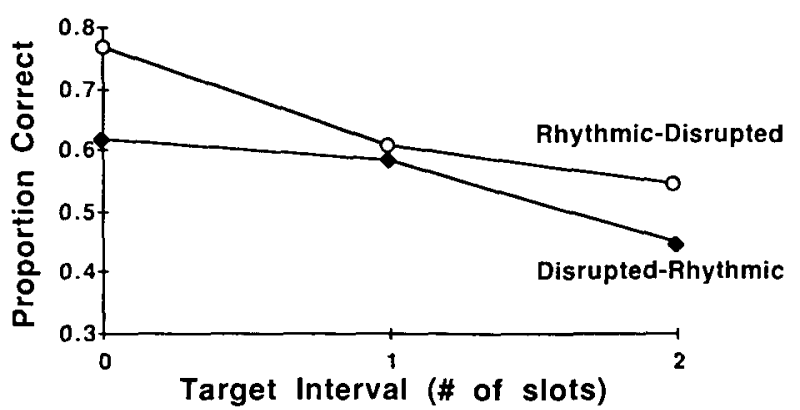

Figure 2. Proportion correct plotted as a function of the size of the target interval, measured by the number of isochronous slot intervals. Performance was better when the rhythmic sequence was presented first, and decreased as the target interval size increased.

\section{DISCUSSION}

Alterations disrupting the isochronous mapping of a rhythmic sequence were more easily detected than were alterations conferring an isochronous mapping on a disrupted sequence. The insertion of a rhythmic anomaly is thus more easily detected than is the removal of one. ${ }^{2}$ This mirrors results obtained for tonal anomalies in melodies (Bharucha, 1984a; Dowling \& Bartlett, 1981) and chord sequences (Bharucha \& Krumhansl, 1983; Krumhansl et al., 1982), and for semantic or pragmatic anomalies in text (Bharucha et al., 1985).

An altered SOA in the comparison is more likely to be confused with the original if it is consistent with the isochronous grid interval than if it is not. The isochronous grid interval is thus abstracted from the standard sequence and incorporated into its representation, as most hierarchical models would suggest (Deutsch, 1983; Deutsch \& Feroe, 1981; Lerdahl \& Jackendoff, 1983; Povel, 1984). When the standard is rhythmic, all the SOAs contribute to the abstraction of an isochronous interval. When the standard is disrupted, all but one contribute. In either case, the representation of the standard includes abstract temporal information. A changed SOA is more likely to be confused with the original if it matches the isochronous grid interval than if it does not.

Subjects were more confident of their correct same responses for rhythmic sequences than for disrupted sequences, suggesting that the disrupted sequences had less stable representations in memory. Clarifying the precise form of the representation of disrupted sequences is beyond the scope of this study. However, several possibilities come to mind. The disrupted sequences, being anomalous in only one SOA, may have been forced into an isochronous mold, resulting in a representation identical to the rhythmic counterpart but slightly less stable. The rhythmic bias observed in imitations of anomalous sequences (see Fraisse, 1982; Povel, 1981) supports this possibility. Alternatively, the disrupted sequence may have been forced into an isochronous mold along with a special tag indicating the presence and nature of the 
anomaly. This mechanism has been proposed for text by Graesser et al. (1979). Yet another possibility is that the grid resolution may have been adjusted once the target interval was encountered, and the entire sequence reparsed on the basis of a grid twice as fine, since the anomaly was constructed by increasing the target interval by half a slot interval. In this case, the economy of the mapping of onsets onto a grid would have been reduced considerably (see Povel, 1984), thereby reducing the stability of the representation.

In conclusion, the addition of a disruptive SOA is more easily detected than is the omission of one. This asymmetry is found in other domains in which we have intuitions of coherence, both musical and propositional, implicating common principles governing the processing of sequences of events we judge to be coherent.

\section{REFERENCES}

Bartlett, F. C. (1932). Remembering. New York: Cambridge University Press.

Bartlett, J. C. (1984). Cognition of complex events: Visual scenes and music. In W. R. Crozier \& A. J. Chapman (Eds.), Cognitive processes in the perception of art. Amsterdam: North-Holland.

BHARUCHA, J. J. (1984a). Anchoring effects in music: The resolution of dissonance. Cognitive Psychology, 16, 485-518.

BHARUCHA, J. J. (1984b). Event hierarchies, tonal hierarchies, and assimilation: A reply to Deutsch and Dowling. Journal of Experimental Psychology: General, 113, 421-425.

BharUCha, J. J., \& KRUMHANSL, C. L. (1983). The representation of harmonic structure in music: Hierarchies of stability as a function of context. Cognition, 13, 63-102.

Bharucha, J. J., Olney, K. L., \& Schnurr, P. P. (1985). Detection of coherence-disrupting and coherence-conferring alterations in text. Memory \& Cognition, 13, 573-578.

Biederman, I., Glass, A. L., \& Stacy, E. W. (1973). Searching for objects in real world scenes. Journal of Experimental Psychology, 97, 22-27.

BLACK, J. B. (1984). Understanding and remembering stories. In J. R. Anderson \& S. M. Kosslyn (Eds.), Tutorials in learning and memory: Essays in honor of Gordon Bower. New York: Freeman.

BOWER, G. H., BLACK, J. B., \& TURNER, T. J. (1979). Scripts in memory for text. Cognitive Psychology, 11, 177-220.

Castellano, M. A., Bharucha, J. J., \& Krumhansl, C. L. (1984). Tonal hierarchies in the music of North India. Journal of Experimental Psychology: General, 113, 394-412.

Cuddy, L. L., Cohen, A. J., \& Miller, J. (1979). Melody recognition: The experimental application of musical rules. Canadian Journal of Psychology, 33, 148-157.

DEUTSCH, D. (1983). The generation of two isochronous sequences in parallel. Perception \& Psychophysics, 34, 331-337.

DEUTSCH, D. , \& FEROE, J. (1981). The internal representation of pitch sequences in tonal music. Psychological Review, 88, 503-522.

Dow LING, W. J. (1978). Scale and contour: Two components of a theory of memory for melodies. Psychological Review, 85, 341-354.

DOWLING, W. J., \& BARTLETT, J. C. (1981). Assimilation of brief atonal melodies to tonal prototypes: Asymmetrical effects on judgment. Paper presented at the 22nd Annual Meeting of the Psychonomic Society, Philadelphia.
Fowler, C. A. (1983). Converging sources of evidence on spoken and perceived rhythms of speech: Cyclic production of vowels in monosyllabic stress feet. Journal of Experimental Psychology: General, 112, 386-412.

FraIsSe, P. (1982). Rhythm and tempo. In D. Deutsch (Ed.), The psychology of music. New York: Academic Press.

Francès, R. (1972). La perception de la musique. Paris: Librairie Philosophique J. Vrin.

Graesser, A. C., Gordon, S. E., \& Sawyer, J. D. (1979). Memory for typical and atypical actions in scripted activities: Test of a script pointer + tag hypothesis. Journal of Verbal Learning \& Verbal Behavior, 18, 319-332.

HANDEL, S., LAWSON, G. R. (1983). The contextual nature of thythmic interpretaton. Perception \& Psychophysics, 34, 103-120.

JoNes, M. R., KIDD, G., \& WETzEL, R. (1981). Evidence for rhythmic attention. Joumal of Experimental Psychology: Human Perception \& Performance, 7, 1059-1073.

KrumhansL, C. L. (1979). The psychological representation of musical pitch in a tonal context. Cognitive Psychology, 11, 346-374.

Krumhansl, C. L., Bharucha, J. J., \& Castellano, M. A. (1982). Key distance effects on perceived harmonic structure in music. Perception \& Psychophysics, 32, 96-108.

Krumhansl, C. L., Kessler, E. J. (1982). Tracing the dynamic changes in perceived tonal organization in a spatial representation of musical keys. Psychological Review, 89, 334-368.

Lehnert, W. G. (1981). Plot units and narrative summarization. Cognitive Science, 5, 293-331.

LERDAHL, F., \& JACKENDOFF, R. (1983). A generative theory of tonal music. Cambridge: MIT Press.

Miller, G. A., \& IsARD, S. (1963). Some perceptual consequences of linguistic rules. Joumal of Verbal Learning \& Verbal Behavior, 2 , 217-228.

PoVEL, D.-J. (1981). Internal representation of simple temporal patterns. Journal of Experimental Psychology: Human Perception \& Performance, 7, 3-18

Povel, D.-J. (1984). A theoretical framework for rhythm perception. Psychological Research, 45, 315-337.

RuMELHART, D. E. (1977). Understanding and summarizing brief stories. In D. G. Bobrow \& A. M. Collins (Eds.), Basic processes in reading: Perception and comprehension. Hillsdale, NJ: Erlbaum.

SCHENKER, H. (1979). Free composition (E. Oster, Ed. and Trans.). New York: Longman. (Original work published 1935)

WINER, B. J. (1971). Statistical principles in experimental design. New York: McGraw-Hill.

YESTON, M. (1976). The stratification of musical rhythm. New Haven, CT: Yale University Press.

\section{NOTES}

1. Mapping onto an isochronous grid does not constitute a definition of a rhythmic sequence, since rhythm is a many-faceted phenomenon (see Fraisse, 1982). According to some theoretical accounts, our thythmic and disrupted sequences may more accurately be termed metrical and ametrical, respectively (see Yeston, 1976).

2. An alternative explanation is that the asymmetry is based on the difference in total duration of the rhythmic and disrupted sequences, the latter being $100-150$ msec longer. This is unlikely since the total duration of each sequence is $2,600-3,900 \mathrm{msec}$, making the difference in duration $1 / 26$ of the total duration.

(Manuscript received December 30, 1985; revision accepted for publication June 30,1986 .) 